\title{
Comunidades virtuales e innovación: propuestas desde la asesoría técnica pedagógica en la escuela telesecundaria
}

\author{
Virtual communities and innovation: proposals from the \\ technical pedagogical advisory in telesecondary school
}

\author{
Karen Bonilla Santamaría \\ Gerson Edgar Ferra Torres
}

\begin{abstract}
RESUMEN
La función de la asesoría técnica pedagógica en la modalidad de Telesecundaria en México es un campo de estudio poco explorado, sobre todo si le agregamos el componente de las tecnologías de la información, comunicación, conocimiento y aprendizaje digitales (TICCAD). En el presente artículo se presentan los resultados de una propuesta de innovación realizada en el marco de la Maestría Innovación en Educación Básica (MIEB) de la Benemérita Escuela Normal Veracruzana "Enrique C. Rébsamen" en Xalapa, Veracruz, México. Con base en la metodología de investigación-acción, se realizó un diagnóstico y valoración de la pertinencia del acompañamiento técnico-pedagógico en una zona escolar de telesecundarias federales a través de la implementación y seguimiento de una comunidad virtual de aprendizaje (CVA), en el cual el trabajo colaborativo se concretó en el diseño de situaciones didácticas con miras a fortalecer la intervención docente en las aulas.

Palabras clave: educación básica, educación no-formal, formación docente, investigación-acción, trabajo en equipo.

ABSTRACT

The function of the pedagogical technical advisory in the Telesecondary modality in Mexico is a field of study quite little explored, especially if we add the component of Information Technology, Communication, Knowledge and Digital Learning (ITCKDL). In this article, the results of an innovation proposal are shown within the framework of the Master's degree in Innovation in Basic Education (MIBE) of the Benemérita Escuela Normal Veracruzana "Enrique C. Rébsamen" in Xalapa, Veracruz, Mexico. Based on the action-research methodology, a diagnosis and assessment of the relevance of the technical-pedagogical accompaniment in a school zone of federal telesecondary schools was carried out through the implementation and monitoring of a virtual learning community (VLC), in which the collaborative work took shape in the design of didactic situations with a view of strengthening the teaching intervention in the classrooms.

Keywords: basic education, non-formal education, teacher training, action-research, teamwork.
\end{abstract}




\section{INTRODUCCIÓN}

En el año de 1968 se estableció en México el primer modelo de educación telesecundaria, el cual surgió de la identificación por parte del gobierno federal de las potencialidades de la televisión como un medio que permitiría hacer llegar el servicio educativo a las zonas alejadas y socioeconómicamente vulnerables. Actualmente la educación telesecundaria representa casi el 50\% de los planteles educativos de secundaria en México, además el $21.1 \%$ de la matrícula escolar se encuentra concentrado en esta modalidad, aglutinando a aproximadamente 73,000 docentes, quienes representan cerca del 18\% del total de la plantilla de educación secundaria (SEP, 2020).

En sus inicios el modelo de telesecundaria se enfocó en difundir contenido educativo homogéneo, desatento de los procesos de enseñanza y aprendizaje acontecidos en las aulas. Sin embargo, en el marco de las tendencias de política educativa internacional vinculadas a la satisfacción de las necesidades básicas de aprendizaje, en 1992 la implementación del Acuerdo Nacional para la Modernización de la Educación Básica (ANMEB) constituyó el instrumento que contribuyó en la concreción de la secundaria en México como el estrato obligatorio del trayecto formativo básico. La telesecundaria repuntó a procesos pedagógicos más completos, bajo los cuales la transmisión televisada cedió protagonismo al manejo de otros materiales educativos, así como a la función del docente como principal guía hacia el logro de los aprendizajes.

Entre los año 2003 y 2004 se gestó la Reforma Integral de Educación Secundaria (RIES), cuyos ideales, de acuerdo con Zorrilla (2004), fueron dirigidos a ampliar la cobertura del servicio educativo, asegurar mejores niveles de logro de los aprendizajes, atender a la diversidad de la población estudiantil, articular coherentemente los tres niveles que conformaban la educación básica, así como transformar las condiciones operativas en los centros educativos orientadas a favorecer el trabajo pedagógico. A pesar de algunos esfuerzos de la federación, la RIES no pudo concretarse al nivel

Karen Bonilla Santamaría. Profesora adscrita a la Supervisión de Escuelas Telesecundarias, número 60, Veracruz, México. Es licenciada en Educación Secundaria con especialidad en Telesecundaria por la Benemérita Escuela Normal Veracruzana "Enrique C. Rébsamen” y maestra en Innovación en Educación Básica. Cuenta con seis años de experiencia como docente frente a grupo de telesecundaria. En el 2017 fue promovida como asesor técnico pedagógico en el área de Pensamiento Matemático, obteniendo nombramiento definitivo en dicha función por los procesos de evaluación del Servicio Profesional Docente en el 2018. Correo electrónico: kbonilla@msev.gob.mx. ID: https://orcid.org/0000-0001-7152-9268.

Gerson Edgar Ferra Torres (autor de correspondencia). Profesor de tiempo completo e integrante del Cuerpo Académico Políticas Públicas y Evaluación Educativa de la Benemérita Escuela Normal Veracruzana “Enrique C. Rébsamen”. Catedrático de la licenciatura en Educación Primaria, de la maestría Innovación en Educación Básica y de la maestría en Competencias Profesionales en la Educación Física de la Unidad de Estudios de Posgrado. Actualmente es jefe del Área de Investigación Educativa en dicha institución. Cuenta con reconocimiento al Perfil ProdeP y es candidato a doctor en Sistemas y Ambientes Educativos por la BUAP. Correo electrónico: gferra@msev.gob.mx. ID: https:/ /orcid.org/0000-0002-0444-9505. 
esperado, sin embargo contribuyó a sentar las bases hacia la búsqueda de igualdad de oportunidades y diversificación de la oferta educativa (Estrada, 2017).

En este escenario, el modelo de telesecundaria (2006) se centró en el reconocimiento de los múltiples contextos escolares, por lo que las adecuaciones curriculares tomaron relevancia. De igual forma se favoreció la incorporación de las tecnologías de la información y la comunicación (TIC) como herramientas para coadyuvar el logro de los aprendizajes; el rol docente adquirió así mayor libertad, y por ende mayor responsabilidad en los procesos didácticos.

En el 2011 se llevó a cabo la Reforma a la Educación Básica, bajo la cual se fortaleció la perspectiva de una articulación curricular entre la educación inicial, preescolar, primaria y secundaria. Posteriormente, en el 2017 se enfatizó en la idea de la escuela como unidad de cambio, con autonomía de gestión y curricular. En este contexto los docentes, de acuerdo con las características y posibilidades del centro educativo, contaron con cierto margen de decisión en cuanto a la distribución de su calendario escolar, así como la implementación de clubes de fortalecimiento académico, de desarrollo personal y social, o de algún otro ámbito que resultara relevante a las necesidades de los estudiantes.

Actualmente, el modelo educativo vigente es la "Nueva Escuela Mexicana", cuyas bases, a nivel discursivo, pretenden dejar de lado el carácter utilitario y eficientista de la educación, inclinándose por la incorporación de los elementos axiológicos, científicos, culturales y artísticos en el favorecimiento del desarrollo integral de las personas. Desde esta perspectiva se concibe al docente como un agente fundamental del proceso educativo, por tanto se reconoce su contribución a la transformación social (LGE, 2019).

Esta aproximación sobre las principales reformas educativas en México describe las constantes transformaciones que impactan en la conceptualización y acción educativa, principalmente de la secundaria como el punto final de la educación básica. Aunque la profundización sobre estas transformaciones queda al margen de los propósitos de este trabajo, es importante considerarlas como un referente hacia la comprensión y focalización de la complejidad del rol docente, reconfigurado continuamente desde los ideales oficiales y en la cotidianidad escolar.

En congruencia con los ideales del Acuerdo Educativo Nacional vigente, recobra relevancia otorgar a las maestras y los maestros del sistema educativo nacional (SEN) el derecho a una mejor formación y una constante actualización (LGSCMM, 2019). En miras a dicho objetivo, se promueve el uso de las TIC, ahora denominadas tecnologías de la información, comunicación, conocimiento y aprendizaje digitales (TICCAD), hacia la digitalización de la educación, capaz de coadyuvar a un aprendizaje continuo y relevante en cualquier espacio y tiempo disponibles (SEP, 2020). 
En definitiva, la tecnología ofrece valiosas oportunidades para la comunicación y el aprendizaje; actualmente su incorporación se encuentra en un periodo de replanteamiento, a raíz del análisis de experiencias llevadas a cabo anteriormente (Red Escolar 1995-2000, Enciclomedia 2001-2012, Programa Habilidades Digitales para Todos 2007-2012, entre otras), cuyos resultados no fueron los esperados debido a una falta de adaptabilidad a las necesidades contextuales de los planteles, así como a la carente capacitación docente sobre el dominio tecnológico y los modelos pedagógicos requeridos para hacer uso efectivo de las TICCAD (SEP, 2020).

La generación de estrategias que resignifiquen el uso de las TICCAD se postula como una pieza angular en los procesos de formación docente. En la modalidad de telesecundaria específicamente, dichas estrategias deben de considerar la diversidad de perfiles profesionales y el modelo pedagógico específico bajo el cual un solo profesor trabaja con los estudiantes todas las asignaturas del currículo, además de revalorizar al profesor y sus prácticas, para transitar de modelos unidireccionales rígidos a otros más flexibles y abiertos a la colaboración profesional (Vaillant, 2016).

En los procesos de formación docente inciden diversas figuras, una de ellas es la supervisión escolar, y específicamente el asesor técnico pedagógico (ATP), quien desde el sistema educativo mexicano es reconocido como un docente con la responsabilidad de brindar a otros docentes asesoría y constituirse como un agente de mejora de la calidad de educación de las escuelas (SEP, 2019).

El ATP se asume como un profesional promotor de estrategias de innovación encaminadas al fortalecimiento de las prácticas pedagógicas, de ahí la importancia de orientar los procesos de asesoría a la creación de nuevas oportunidades formativas y ambientes de aprendizaje innovadores, donde las TICCAD representen para el profesorado auténticas oportunidades de aprendizaje y desarrollo profesional.

Sin embargo, el ATP surge como un elemento de apoyo a las labores de la supervisión, su margen de actuación se condiciona a una estructura organizacional vertical, estando sujetos a las predisposiciones de la política educativa en turno. El asesoramiento se ha constituido como un instrumento para resolver necesidades de las administraciones educativas (Rivera y Martínez, 2015), limitando en cierta forma la autonomía del asesor en los procesos de mejora.

El ATP como integrante de la supervisión escolar colabora con el supervisor, directores y colegas manteniendo como meta común lograr el desarrollo integral de los alumnos. No obstante, el número reducido de asesores en las zonas escolares en relación con el número de escuelas a atender, aunado a la carga administrativa, complejiza la realización de proyectos sistemáticos de intervención particulares a cada centro escolar, por lo que, a pesar de que se reconocen como necesarios, los 
apoyos a las escuelas no siempre logran el impacto pretendido como facilitadores del aprendizaje para la eficacia escolar (Segovia, 2010).

Para el ATP puede resultar complejo impulsar procesos de mejora, sobre todo porque, aunque forma parte de la estructura educativa, es un agente externo a los centros escolares, con un ambiguo margen de actuación como promotor de cambios educativos, limitándose por lo regular a brindar capacitación sobre algún programa federal o estatal específico, a visitar escuelas focalizadas, observar sesiones de clase para brindar retroalimentación a los docentes, organizar el trabajo con directivos y docentes para la realización de las sesiones de Consejo Técnico Escolar (CTE), entre otras funciones. A pesar de que estas actividades son valiosas y necesarias, por lo regular se quedan cortas en potencializar transformaciones sustanciales en las prácticas pedagógicas que generen en los estudiantes más y mejores oportunidades de aprendizaje.

Actualmente, el ATP se enfrenta a la necesidad de realizar una reconfiguración de sí mismo ante las condiciones de la llamada "sociedad del conocimiento", de su entorno laboral y su práctica profesional (Cruz-Abarca, Bazán-Ramírez, Taracena-Ruiz y Castellanos-Simons, 2016). Es prioritario que el ATP impulse nuevos mecanismos para que paulatinamente, en conjunto con otros agentes, puedan desplegarse medidas hacia el fortalecimiento de la calidad educativa y el desarrollo colectivo, como aspectos prioritarios a atenderse desde la supervisión escolar (Rivera, 2017).

Un reto importante del ATP del nivel de telesecundaria es revitalizar su propia función como un agente de cambio y de transformación que contribuya a potenciar el trabajo colaborativo, el aprovechamiento de liderazgos pedagógicos y la incidencia en identificar mejores prácticas educativas para el logro de los aprendizajes.

\section{CONTEXTUALIZACIÓN DE LA INNOVACIÓN Y LA INVESTIGACIÓN}

El medio que enmarcó la propuesta se encuentra ubicado en una Zona Escolar de Telesecundarias de sostenimiento federalizado, en el estado de Veracruz, México. Dicha zona se conforma por 16 planteles escolares, dos de los cuales se encuentran en contexto urbano, mientras el resto se ubica en contextos rurales con alto grado de marginación. Las condiciones de infraestructura entre los planteles son sumamente variables; algunos de estos cuentan con servicios de cómputo, cobertura de internet, domos y canchas, mientras otros carecen de equipamientos y servicios para el desarrollo óptimo de las actividades.

Para mayo del 2018, momento en que se inició la investigación, la plantilla de personal estaba conformada por noventa docentes frente a grupo, cinco de ellos además desempeñaban la función de directores comisionados; once directores efectivos 
y diez apoyos administrativos, que en conjunto atendían a cerca de 1,330 alumnos distribuidos en los 16 planteles.

Cada escuela bajo el liderazgo de los directores ejercía su autonomía al establecer los objetivos, metas y acciones de su Programa Escolar de Mejora Continua (PEC), elaborado al inicio de ciclo durante la fase intensiva del CTE. Este programa parte de la identificación de las principales problemáticas que el colectivo delimita en relación con ocho ámbitos relacionados con el aprendizaje de los alumnos, las prácticas docentes, la relación escuela comunidad, el liderazgo directivo y la descarga administrativa. A través del desarrollo de las sesiones ordinarias, los profesores realizaban los ajustes y precisiones al PEC con base en la identificación de los avances obtenidos.

En lo relativo a la organización y funcionamiento de la supervisión escolar, esta se integraba de un supervisor, tres ATP y cuatro apoyos administrativos. De manera general, la supervisión se encargaba de hacer llegar a los centros educativos, principalmente a través de los directores, la información indicada por la autoridad educativa local respecto a programas, lineamientos sobre procesos de registro y certificación de alumnos, así como los materiales y pautas para el desarrollo de los Consejos Técnicos de zona y escolares.

La detección de la problemática se realizó durante el periodo comprendido entre los meses de enero y mayo del 2018, principalmente a través de herramientas de corte cualitativo, como las siguientes:

- Formularios en línea a directores escolares: implementados para recabar necesidades de intervención técnico-pedagógica que identifican en los planteles de la zona escolar. En los resultados de la aplicación, la mayoría coincidió en la importancia de brindar a los profesores orientaciones para la planeación de las clases, materiales didácticos y estrategias de evaluación.

- Notas de campo: recabadas en las sesiones del Consejo Técnico de zona (supervisión con directores) y en sesiones ordinarias de Consejo Técnico escolar (directores y docentes). Se identificó que, de manera general, los docentes interactuaban en torno a problemáticas generales de los alumnos, por ejemplo: coincidían en la importancia de "desarrollar el pensamiento lógico-matemático" o en "apoyar a los alumnos en la comprensión de textos", incluso planteaban algunas estrategias como "cafés literarios", "círculos de lectura", "talleres de matemáticas", no obstante, pocas veces ahondaban en el tipo de intervención docente requerida para estas estrategias o delimitaban con exactitud el aprendizaje esperado involucrado en las mismas, es decir, carecían de precisión en la definición de los objetivos educativos a alcanzar y en la intervención pedagógica requerida para tal efecto. 
- Observaciones de clase: realizadas en diversas escuelas para registrar semejanzas y diferencias en la intervención didáctica desarrollada en las aulas. Se constató la existencia de una amplia diversidad de intervenciones en el aula. Se apreciaron estrategias didácticas de distinta naturaleza que los profesores realizaban en el tratamiento cotidiano de los contenidos. La mayoría de estas intervenciones quedaban limitadas a cada salón de clase, sin una socialización y/o retroalimentación posterior entre colegas.

Con base en lo anterior, se valoró como área de oportunidad la limitada comunicación entre los docentes en referencia a las prácticas didácticas cotidianas. Dada la diversidad de perfil profesional, edad y antigüedad en el servicio del profesorado, se tornó oportuno brindar marcos de actuación que facilitaran la colaboración a través de sistemas de apoyo basados en el co-aprendizaje.

Desde la óptica planteada, partiendo de la complejidad de operación y seguimiento a las prácticas docentes desde la supervisión escolar, se ratificó que esta, y especialmente el ATP, más allá de intentar brindar un asesoramiento personalizado a cada docente, tendría además que coadyuvar a situaciones y espacios desde la innovación en el uso de las TICCAD para poner en contacto la múltiples perspectivas y formas de actuación en el aula, incluso entre centros escolares diversos y físicamente distantes, atendiendo así la problemática: escasa colaboración docente para el fortalecimiento de la intervención didáctica cotidiana.

Una vez definida la problemática a atender, se procedió a delimitar los alcances de la investigación mediante el objetivo general: "Promover el trabajo colaborativo a través de una comunidad virtual de aprendizaje (CVA) docente para fortalecer la intervención didáctica en telesecundaria”, y los siguientes objetivos específicos: 1) Reconocer la potencialidad de la CVA para promover el trabajo colaborativo; 2) Identificar el impacto de la CVA en la intervención didáctica en telesecundaria, y 3) Comprender la transformación de la práctica del agente promotor de la innovación con relación a la función de asesoría técnica pedagógica en telesecundaria.

\section{REVISIÓN DE LITERATURA}

Emprender una propuesta con miras a la innovación conduce necesariamente a delimitar, en un primer momento, qué se entiende por “innovación” y desde qué perspectiva o paradigma la abordaremos. En este sentido, el esbozo de esta propuesta se cimentó principalmente en las aportaciones de Rivas (2000), quien entiende a la innovación educativa como: "Proceso de incorporación de algo nuevo en el sistema de la institución escolar, cuyo resultado es la modificación de su estructura y operaciones, de tal modo que mejora sus efectos en orden al logro de los objetivos educativos" (p. 27). 
Solís, Hernández, Téllez, Cuenca y Guerrero (2007) nos señalan que la innovación implica un cambio que deriva en mejorar cualitativamente los aprendizajes educativos, mientras que García-Peñalvo (2015) también la identifica como un cambio eficaz, eficiente y dependiente del contexto en que se desarrolla. Carbonell (2000) define a la innovación como "una serie de intervenciones, decisiones y procesos, con cierto grado de intencionalidad y sistematización, que tratan de modificar actitudes, ideas, culturas, contenido, modelos y prácticas pedagógicas" (p. 15).

De las aproximaciones planteadas, en esta propuesta se determina que la innovación conlleva un cambio en un contexto educativo determinado, el cual condiciona su naturaleza y finalidades. Desde esta perspectiva, la innovación educativa podría entenderse como: una modificación sustancial de algún aspecto clave de la realidad escolar, con la finalidad de elevar y mejorar los aprendizajes de los agentes involucrados.

Desde un posicionamiento definido respecto a la innovación educativa, se analizaron los posibles alcances en torno a la reconfiguración de la asesoría técnica pedagógica para promover espacios de desarrollo profesional. En este sentido, se partió de reconocer la complejidad del ejercicio docente, impactado frecuentemente por el paradigma o política educativa en turno; las realidades contextuales en las que se desarrolla y por la singular intencionalidad de cada docente (Fullan y Hargreaves, 1999).

Knowles, considerado el padre de la andragogía como ciencia encargada de estudiar los procesos de aprendizaje de los adultos (en este caso los docentes), concibe a la experiencia como la fuente más valiosa de educación para el adulto aprendiz, bajo un enfoque que conste de situaciones pertinentes, no de temas (Knowles, Holton y Swanson, 2006). Por otra parte, Tardif (2004) plantea que los profesores son poseedores de saberes de distinta índole, que van desde su formación profesional, aquellos de carácter disciplinario, curricular y experiencial; amalgamados y redimensionados continuamente desde la singular personalidad e identidad de cada profesor. De esta manera, la elección de las situaciones pertinentes es en sí uno de los mayores desafíos para los formadores y asesores de docentes, quienes se enfrentan a la tarea de promover aprendizajes profesionales en función de la movilización de los saberes docentes.

El campo que aquí ocupan se refiere específicamente al aprendizaje docente, noción que podría equipararse al desarrollo profesional (Vaillant y Marcelo, 2015) en el momento en que se asume al profesor como el especialista en la enseñanza, y por ende como uno de los responsables directos del aprendizaje de los estudiantes. Por ello es prioritario comprender y repensar las estrategias para impulsar el aprendizaje docente de tal forma que estas intervenciones atiendan sus necesidades con resultados plausibles en las aulas.

Cabe puntualizar que el tipo de aprendizaje pretendido va más allá del mero ámbito técnico o empírico, por el contrario, pretende favorecer la externalización de 
aquellas creencias o teorías personales sobre la enseñanza y el aprendizaje, profundamente asumidas por cada docente, que condicionan, por lo regular sin una conciencia crítica, la práctica educativa (Echeverría, Municio, Rubiños, De la Cruz, Ortega y Sanz, 2006). Es así como el llamado "currículo oculto" debería comprenderse como instrumento y a la vez objeto de las estrategias hacia la transformación escolar.

En definitiva, el aprendizaje docente parte de la externalización y movilización de los saberes docentes puestos en marcha en la dinámica de clase, además debe estar fuertemente ligado con su práctica profesional más importante: la enseñanza. He aquí una complejidad: ¿De qué manera puede coadyuvarse a dicho aprendizaje? En este punto se ha de tener en cuenta que el aprendizaje está asociado directamente a la acción (Senge, 2012), una acción apremiante en cada decisión del profesor, en cada interacción con los estudiantes, en cada cambio de asignatura, en cada momento de la llamada "cotidianidad escolar".

La reflexión sobre la propia práctica conforma la principal línea formativa de diversas tendencias relacionadas a la formación de profesores (Breda, Font y PinoFan, 2018). No obstante, antes de pretender potenciar niveles profundos de reflexión docente, habría que apoyar en la construcción de espacios que permitan sistematizar la práctica, de tal manera que los profesores cuenten con plataformas para organizar e interpretar sus acciones didácticas.

A la interpretación de la actuación que el docente realiza en el aula al trabajar un contenido en concreto le llamaremos "intervención didáctica". Este concepto está experimentando un auge importante, principalmente en el área de la enseñanza matemática, sin embargo, en el proyecto aquí planteado se le emplea como una herramienta igualmente valiosa en el análisis didáctico de otras disciplinas.

La noción de intervención didáctica referida en la propuesta es el resultado de construcciones parciales derivadas de la literatura actual, principalmente aquellas vinculadas a la teoría de la idoneidad didáctica (Godino, Batanero, Rivas y Arteaga, 2013) y de las situaciones didácticas (Brousseau, 2007) que apoyan en la identificación de múltiples dimensiones (epistémica, ecológica, afectiva, interaccional) que confluyen en el aula, sobre las cuales el profesor toma decisiones antes, durante y después de una sesión.

Es de tomar en cuenta que la sistematización de la propia intervención didáctica puede resultar una tarea poco productiva si el docente se basa en su criterio como único e inequívoco. Por ello, en este contexto, el aislamiento se identifica contraproducente, al desorientar al docente, conduciéndolo a la pérdida de su intencionalidad (Fullan y Hargreaves, 1999). En consecuencia, cualquier estrategia que pretenda incidir en la intervención didáctica debería contemplar la colaboración efectiva entre colegas. 
El trabajo colaborativo se perfila como una estrategia clave hacia el desarrollo profesional docente (Vaillant, 2016). Aplicada adecuadamente puede resultar una poderosa herramienta en el intercambio y provisión de recursos para aprender en y desde la práctica (Krichesky y Murillo, 2018), razón por la cual los sistemas formativos docentes deberían apostar por intervenciones que favorezcan la colaboración y así poder comprender de una forma más precisa cómo se aterrizan los procesos derivados de dicha estrategia.

En este tenor, la conformación de comunidades virtuales se proyecta como una alternativa que permite la comunicación asincrónica y el aprovechamiento efectivo de múltiples herramientas disponibles en el ciberespacio con el fin de colaborar en la resolución de situaciones profesionales comunes entre sus participantes.

En el plano internacional, las prácticas virtuales en general comienzan a tener un auge importante en el mapeo de las tendencias en innovación educativa, tal como fue constatado en el III Congreso Internacional sobre Aprendizaje, Innovación y Competitividad CinAic 2015. Aunque su expansión es más vertiginosa en el ámbito empresarial, paulatinamente se está incorporando a programas formativos al facilitar el trabajo a distancia, optimizando tiempos y costos de desplazamiento (GarcíaPeñalvo, 2015).

Es así como la virtualidad, aplicada al terreno de profesionalización, podría materializar oportunidades para hacer un uso más eficiente de las TICCAD en trayectos formativos con elementos de innovación para promover una colaboración profesional asincrónica y la optimización de recursos materiales y humanos, considerando que una comunidad virtual se constituye en un espacio para el aprendizaje docente, en tanto "busca transformar las prácticas pedagógicas y la organización habitual de las instituciones educativas formales, al mismo tiempo que moviliza los recursos ajenos a estas poniéndolos al servicio de la educación y de la formación de las personas" (Gairín, 2006, p. 4).

De esta forma, el proyecto se basó en incorporar una comunidad de aprendizaje virtual (CVA) con la intención de reconfigurar el empleo de las TICCAD en los espacios de colaboración docente, desde la asesoría técnica pedagógica en telesecundaria. La comprensión de CVA aquí desarrollada partió de las siguientes aportaciones.

Rheingold (1993) define a las comunidades virtuales como "agregaciones sociales que emergen de la Red cuando un número suficiente de personas entabla discusiones públicas durante un tiempo lo suficientemente largo, con suficiente sentimiento humano, para formar redes de relaciones personales en el ciberespacio" (p. 5).

Por su parte, Gairín (2006) la define como "un grupo de personas que aprende conjuntamente, utilizando herramientas comunes en un mismo entorno" (p. 44); Pazos, Pérez y Salinas (2001) consideran a las comunidades virtuales de aprendizaje 
como "ambientes basados en la Web, que agrupan personas relacionadas con alguna temática específica” (p. 2).

A partir de los elementos de las definiciones presentadas, en el marco de la naturaleza de la propuesta innovadora, una comunidad virtual de aprendizaje podría entenderse como: un grupo de personas que aprenden colaborativamente en un entorno virtual, empleando recursos comunes para el logro de objetivos vinculados a su desarrollo profesional.

La CVA brinda matices particulares a esta estrategia formativa al involucrar a los participantes en el uso de la tecnología en beneficio de la transformación de la práctica, a nivel individual y colectivo. El trabajo colaborativo en entornos virtuales se comprende como "el conjunto de métodos de instrucción y entrenamiento apoyados con la tecnología, así como de las estrategias para propiciar el desarrollo de habilidades mixtas (aprendizaje y desarrollo personal y social) donde cada miembro del grupo es responsable tanto de su aprendizaje como el de los restantes miembros del grupo" (Lucero, 2003, p. 4).

El funcionamiento de una CVA busca brindar a los profesores la oportunidad formal de trabajar colaborativamente aspectos e insumos relevantes de su práctica diaria, tales como situaciones didácticas, materiales y estrategias de evaluación; entablar discusiones en torno a temáticas pertinentes a sus contextos y necesidades. El espacio en cuestión potencia el trabajo colaborativo al brindarles a los participantes posibilidades de proyectar su visión sobre la tarea educativa, tanto a nivel discursivo como práctico.

En este sentido, el papel del asesor en ambientes de colaboración profesional demanda la organización y gestión de actividades vinculadas a la instauración de estos espacios. En el proyecto aquí abordado, la asesoría técnica pedagógica en pensamiento matemático de una zona de telesecundarias, se asumió como el agente hacia la innovación a través del uso efectivo de las TICCAD en la organización y potenciación de una CVA, dirigida a la movilización de prácticas y perspectivas docentes que pudieran reestructurar la intervención didáctica en las aulas, mediante el trabajo colaborativo sobre situaciones concretas.

\section{Metodología}

El contexto situacional y objetivos del proyecto se sitúan dentro del paradigma cualitativo de la investigación, con énfasis en la investigación-acción participante. De acuerdo con Hernández, Fernández-Collado y Baptista (2014), se cumplen los siguientes criterios: se trata de una problemática de una comunidad que es necesario resolver y plantear un cambio; la problemática es parte de un grupo o comunidad; se 
utilizaron técnicas como grupos de trabajo, entrevistas, foros de discusión, reuniones, y se planteó un diagnóstico y un programa o proyecto para resolverlo.

El método científico práctico de la investigación-acción (Álvarez-Gayou, 2003) sugiere que su implementación al menos cumpla la mayoría de los siguientes criterios: el estudio de prácticas locales o de la comunidad, desarrollo y aprendizajes de los participantes, implementación de un plan de acción y finalmente el liderazgo del investigador o de un grupo de colaboración. En la figura 1, análogamente, se reconstruye la metodología:

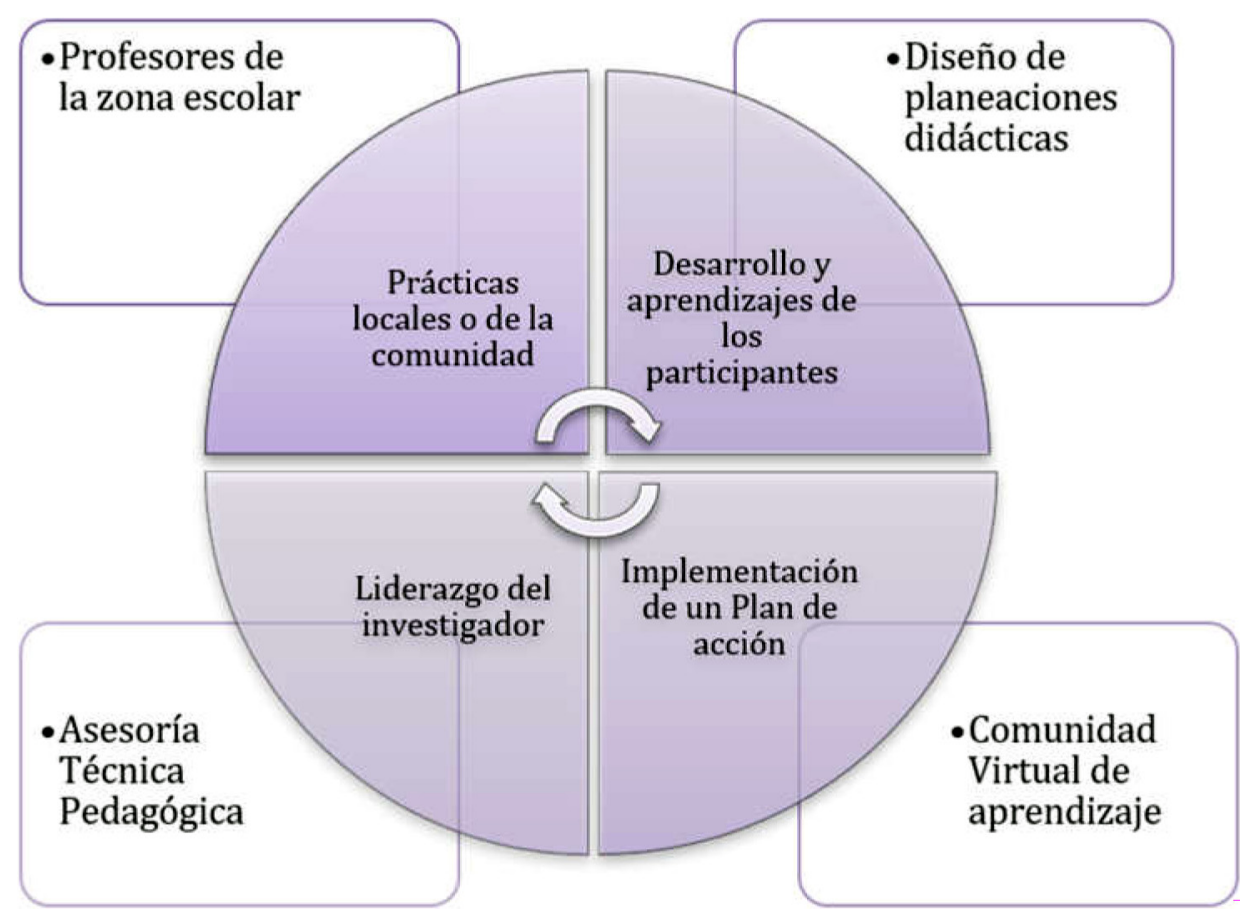

Figura 1. Desarrollo de la propuesta con base en la adaptación análoga de los criterios sugeridos por Álvarez-Gayou (2003) para la implementación del método científico práctico de la investigación acción.

Fuente: Elaboración propia.

A partir del enfoque de investigación-acción, desde el modelo propuesto por Eliott (2000) se desarrolló el proyecto como el resultado de un proceso espiral que parte de una idea general, el reconocimiento de la situación, la planificación, hasta el desarrollo de una primera etapa de acción, a partir de la cual se realiza una revisión de los logros obtenidos desde distintas aristas del plan a fin de hacer los ajustes o restructuraciones pertinentes que reorientan las acciones a desarrollar durante las etapas subsecuentes.

La investigación-acción refiere un análisis de carácter cualitativo, continuo y sistemático que nos acerque al reconocimiento del nivel de logro alcanzado respecto 
a los objetivos propuestos a través del empleo de diversos insumos, para ello ha de tenerse en cuenta la recopilación organizada de la información, y así validar epistemológicamente la acción del investigador (Cabrera, 2005); además, de acuerdo con Abero, Berardi, Capocasale, García y Rojas (2015), hablar de investigación-acción supone referir a una nueva concepción de entender a la institución escolar, el proceso de enseñar y aprender, así como la formación del profesorado y el desarrollo profesional docente.

\section{El proceso de planeación}

La primera actividad realizada fue la selección del espacio virtual que fungió como el medio hacia la concreción de la CVA, de tal manera que su implementación resultase factible y accesible a la totalidad de la planta docente de la zona escolar. Como parte del registro de usuarios institucionales, la Secretaría de Educación de Veracruz (SEV) formalizó el uso de cuentas de correo electrónico con un dominio propio (msev. gob.mx). El registro de estas cuentas brinda a los usuarios la obtención gratuita de Microsoft 365, que consta de un conjunto de 16 aplicaciones para la realización de actividades de distinta naturaleza (servicio de correo electrónico, block de notas, elaboración de lienzos interactivos, creación de cuestionarios, entre otras). Explorando las aplicaciones disponibles, se detectó la posibilidad de optimizar el uso de Microsoft 365 en la implementación de la CVA, a través de la aplicación Microsoft Teams, la cual se describe como:

Un área de trabajo en Office 365 centrada en el chat que ayuda a los miembros a ser más productivos. Sirve como centro para el trabajo en equipo, ya que ofrece acceso instantáneo a conversaciones de chat, contenido y herramientas de Office 365 en una sola área de trabajo. SharePoint y OneNote están integrados y los miembros del equipo pueden trabajar en documentos de Office desde la aplicación. Aparte de los chats, Microsoft Teams también admite videollamadas y reuniones. Los miembros del equipo pueden colaborar fácilmente con varios equipos y buscar contactos, chats y archivos en cualquier momento [Microsoft, 2019, p. 1].

Se recuperaron las aportaciones de Boneu (2007) respecto a la pertinencia de considerar un entorno virtual favorable para el aprendizaje, bajo las siguientes características:

- Interactividad: consigue que el usuario de la plataforma tenga conciencia de su protagonismo.

- Flexibilidad: las funciones facilitan la adaptabilidad de actividades acordes a los objetivos de trabajo implementado.

- Escalabilidad: capacidad de funcionar tanto con un número pequeño como grande de personas (hasta 10,000 miembros). 
- Estandarización: el formato de colaboración involucrado optimiza el uso de una aplicación de gran cobertura, en consecuencia, es potencialmente trasladable a otros contextos escolares, con sus respectivos matices y particularidades. La implementación se organizó en tres etapas: preparación, colaboración y aplicación, con una duración aproximada de 12 semanas. La etapa de preparación consistió en identificar las temáticas y situaciones que pudieran fungir como articuladores del trabajo desarrollado en la CVA; en este caso dichas situaciones se definieron atendiendo a los siguientes criterios:

a) Su relevancia en las prácticas didácticas cotidianas.

b) Su factibilidad de propiciar la colaboración profesional, permitiendo la interacción de prácticas docentes.

Se seleccionó la realización de planeaciones didácticas como una actividad potencialmente significativa a desarrollar en la comunidad virtual, al posibilitar la manifestación de decisiones y cursos de acción que los docentes consideran efectivos en el manejo de las situaciones en el aula. La planeación didáctica se perfiló como una estrategia que apoyaría al profesor en hacer más efectivas dichas decisiones para el logro de los objetivos en pro del aprendizaje de los estudiantes (Salinas, Nevárez y Torres, 2014). Desde una perspectiva socioformativa, la planeación didáctica es en sí la concreción de un proceso complejo, consciente y deliberado que realiza el profesor en lo que, desde su enfoque personal, sería el mejor escenario para el aprendizaje de los estudiantes (Brito-Lara, 2017). Es así como esta herramienta pedagógica, en apariencia simple, materializa sutilmente a una serie de concepciones y saberes del profesor respecto a la enseñanza.

Ante la diversidad, amplitud y dispersión de la población objetivo de la propuesta, se planteó el uso del formulario en línea, utilizando la aplicación Microsoft Forms, con la finalidad de optimizar la organización y el planteamiento del proceso de planeación didáctica como detonante hacia la colaboración profesional. La aplicación del formulario a docentes permitiría conocer los elementos que contemplan en el diseño de las planificaciones, así como las asignaturas en las que ellos consideran tener mayores fortalezas didácticas.

Los resultados del formulario se emplearían para organizar equipos de trabajo para diseño de planeaciones didácticas de tal manera que los integrantes (docentes a cargo de un mismo grado, pero de diferentes escuelas) compartieran estrategias, materiales, recursos, instrumentos de evaluación, pautas de intervención, entre otros elementos que, en su conjunto, constituyeran líneas para el diálogo profesional.

Para la segunda etapa, denominada de colaboración, se conformarían equipos de trabajo de tres integrantes quienes harían uso de las herramientas de la aplicación virtual para editar documentos, hacer reuniones en línea, compartir recursos, etc., 
en la realización de planeaciones didácticas. Los ATP de la supervisión escolar proporcionaron una rúbrica para orientar el diseño de las planeaciones, considerando los siguientes elementos: referentes y elementos curriculares, diseño de actividades articuladas por momentos, organización de los estudiantes, materiales impresos y recursos didácticos, recursos digitales de apoyo, e instrumentos y estrategias de evaluación-retroalimentación.

Además de la rúbrica, los asesores brindarían apoyo virtual y presencial a los equipos en el manejo de la aplicación, así como también auxiliares para el planteamiento didáctico desde el enfoque pedagógico de cada asignatura.

Finalmente, la etapa de aplicación se definió para la conformación de un repositorio (dentro de la aplicación Microsoft Teams) de planificaciones didácticas de las asignaturas de Español, Matemáticas, Ciencias, Historia, Formación Cívica y Ética e Inglés, de tal forma que cualquier docente de la zona escolar pudiera acceder al mismo y seleccionar una o más planeaciones, preferentemente eligiendo aquellas en cuya elaboración no participó.

Los ATP realizarían al menos una visita de observación de clase a un grupo muestra conformado por cinco docentes, de manera aleatoria. Las observaciones de clase se apoyarían de un instrumento para focalizar aspectos relevantes de la intervención didáctica, congruentes a su vez con la rúbrica de planeación didáctica previamente proporcionada. El instrumento en cuestión consistió en una rúbrica sintética que contempló tres niveles de desempeño (insuficiente, suficiente y satisfactorio) en el análisis de los siguientes aspectos de la intervención didáctica:

- Planeación didáctica.

- Recursos didácticos.

- Ambiente de aprendizaje.

- Estrategias didácticas.

- Estrategias e instrumentos de evaluación del aprendizaje.

\section{E1 proceso de seguimiento y evaluación}

El seguimiento a las actividades fue desplegado desde la premisa de que el aprendizaje tiene una connotación altamente social. De dicha premisa se deriva el supuesto de que una comunidad de aprendizaje virtual, al promover el diálogo y la comunicación puede propiciar aprendizajes traducidos en la mejora profesional por parte de sus participantes. Como resultado de este proceso se determinaron como categorías de análisis el trabajo colaborativo y la intervención didáctica, cuya recolección de datos se llevó a cabo principalmente a través de instrumentos de observación participante y no participante. 
La categoría 1, Trabajo colaborativo, se refiere a la interdependencia de un grupo hacia el logro de un objetivo de aprendizaje en común. Con base en las aportaciones de Johnson, Johnson y Holubec (1999) sobre los componentes esenciales para el trabajo colaborativo, se establecieron las siguientes subcategorías de análisis: contribución de conocimiento, interdependencia positiva y trabajo y habilidad para compartir con los demás.

Con respecto a la categoría 2, Intervención didáctica, se vincula con el actuar del docente en el aula al trabajar un contenido en particular. En congruencia con el modelo de la idoneidad didáctica y de los criterios para hacer la caracterización del grupo muestra, las subcategorías de análisis fueron las siguientes: planeación didáctica, recursos didácticos, ambiente de aprendizaje, estrategias didácticas y estrategias e instrumentos de evaluación del aprendizaje.

El diseño y validación de los instrumentos utilizados para la etapa de seguimiento y evaluación partió de la elaboración de una matriz de doble entrada en donde se establecieron, a partir de los objetivos de la propuesta, aquellos que de acuerdo a las dimensiones del estudio fueron los más pertinentes. La validación de los mismos fue del tipo de contenido, ya que se recuperó información de la revisión de literatura y de documentos oficiales. En este sentido, el análisis cualitativo de contenido busca resultados a través de un procedimiento circular de interpretación de textos que, según el enfoque, puede tener como finalidad la construcción de una teoría, la evaluación de unos supuestos teóricos, la tipologización del material encontrado y la estructuración del contenido a través de categorías (Gehrig, Palacios, Blesa, Cobo, García, Muñoz y Rodes, 2014, p. 51).

Tomando en cuenta que el periodo de aplicación de la propuesta fue relativamente breve, se delimitaron únicamente dos ciclos como cortes parciales para el seguimiento de los alcances del proyecto, tal como se puede apreciar en la figura 2.

El primer ciclo se centró en interpretar la forma como se aterrizó la caracterización didáctica del grupo muestra, la organización de equipos para el trabajo, la presentación del proyecto con directivos, así como la elaboración colaborativa de planeaciones didácticas dentro de la aplicación Microsoft Teams. Atendiendo a las categorías de análisis, se implementaron los siguientes instrumentos de seguimiento:

1. Formulario de autoevaluación en línea: diseñado para explorar, a través de siete ítems, la apreciación de la totalidad de la población participante (105 docentes) respecto al nivel de colaboración que manifiesta al interior de los equipos de trabajo, en relación con las subcategorías del trabajo colaborativo, así como en la exploración de las situaciones que desde su perspectiva impactaron en su participación. Bajo este enfoque, la autoevaluación permite al participante identificar qué habilidades desarrolla correctamente y cuáles no y por qué motivo (Núñez y Urquijo, 2012), de tal forma que pueda mejorar como colaborador. 


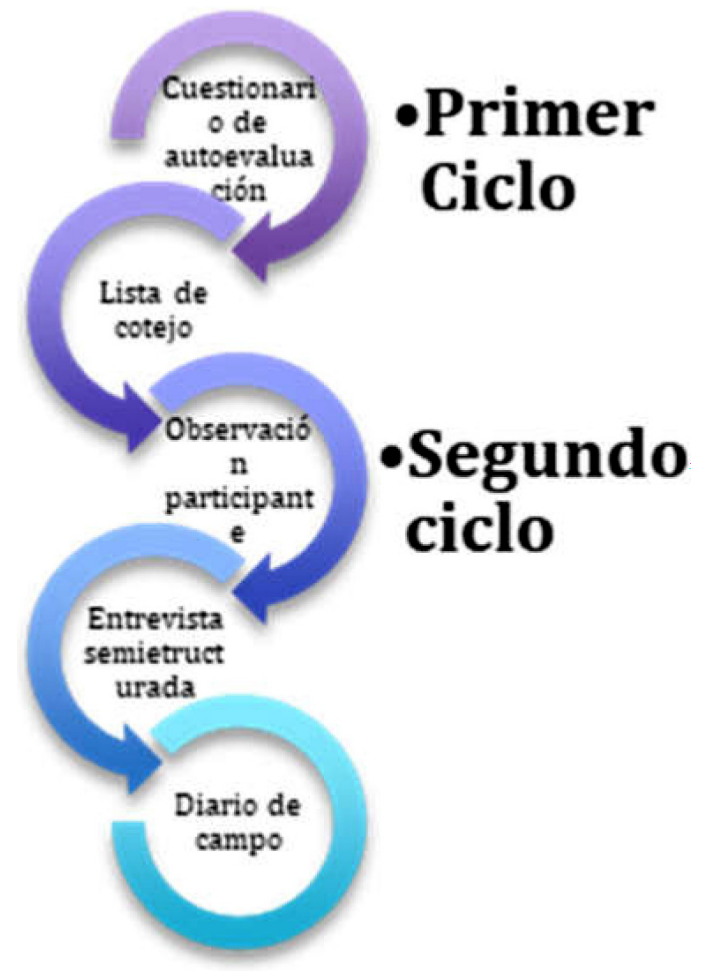

Figura 2. Ciclos de evaluación de la propuesta. Fuente: Elaboración propia.

2. Lista de cotejo: Este tipo de instrumento presenta, conforme con las directrices de una actividad o tarea, una enumeración de conductas, cualidades o características esperables/observables que se evalúan mediante una marca que indica la ausencia o presencia de una conducta o rasgo (Grupal, 2015).

La lista de cotejo se planteó en la determinación del porcentaje de planeaciones subidas a los archivos de la aplicación. El número total de planeaciones disponibles en el repositorio referenciaron el nivel de concreción del trabajo colaborativo en un producto específico, principal insumo para la posterior aplicación en las aulas de las propuestas didácticas elaboradas en la comunidad virtual.

El segundo ciclo se enfocó en conocer las apreciaciones de los docentes respecto al impacto de aplicar en el aula las propuestas didácticas elaboradas por otros profesores, para lo cual se utilizaron los siguientes instrumentos:

1. Rúbrica de observación de clase: tal como su nombre lo sugiere, apoya en la realización de una observación no participante al grupo muestra, para apreciar la actuación del docente y su interacción con los estudiantes (Castañeda, 2018).

2. Entrevista semiestructurada: planteada con el propósito de ahondar en las percepciones respecto a la aplicación de las planeaciones en la intervención. Se apoyó en una guía de preguntas flexibles a precisiones y ajustes con el fin 
de ampliar la información proporcionada por el entrevistado (Hernández, Fernández-Collado y Baptista, 2007) y así contar con mayores elementos en la comprensión de dichas apreciaciones.

3. Diario de campo: referido como un instrumento de registro anecdótico, continuo y acumulativo desde la impresión del investigador (Quintana, 2006) que puede ser objeto de un análisis teórico sobre la práctica. El diario de campo (DdC) representó el instrumento del seguimiento personal cuyo objeto fue proporcionar las bases para la comprensión del papel del ATP dentro de la propuesta. El diario dio soporte a un registro sistemático con la finalidad de enfocar la atención en aspectos relevantes en los distintos procesos involucrados en la instauración de la CVA.

\section{Resultados}

Los resultados del estudio se clasificaron en tres dimensiones que permiten evaluar el cumplimiento de los objetivos propuestos: trabajo colaborativo, intervención didáctica y el agente promotor de innovación.

Sobre el trabajo colaborativo, la elaboración de planeaciones cumplió con la función de potenciar procesos hacia la externalización de saberes y experiencias de los participantes, apegándose así a los lineamientos del modelo instruccional requerido en el diseño didáctico de ambientes virtuales de aprendizaje al impulsar la interacción dinámica para la confrontación de conocimientos (Herrera, 2006).

Los datos obtenidos del formulario de evaluación en línea arrojaron que, en todas las subcategorías, el 73\% de los autoevaluados se ubicó en un nivel de desempeño 3, es decir, consideró:

- Contribuir al trabajo realizando propuestas que favorecen el logro de la meta.

- Alcanzar la meta si todos los demás colaboran y que su esfuerzo le beneficia al grupo.

- Demostrar y expresar sensibilidad hacia los demás alentándolos a participar.

- Contribuir con información y habilidades sin tener que pedírselo.

- Participar voluntariamente en realizar cambios.

Con referencia a los factores que los profesores creen que propiciaron o limitaron su participación, se destacó que valoran la contribución de otros en el nivel de participación que ellos demuestran. No obstante, el tiempo como factor en contra también fue una respuesta constante, además de la falta de organización y comunicación al interior de los equipos. He aquí la presencia de algunos de los inconvenientes que representa a los usuarios trabajar colaborativamente en entornos virtuales, sobre todo si estos últimos van más allá del solo intercambio de información o puntos de vista sobre un tema determinado y en lugar de ello pretenden generar productos concre- 
tos que requieren necesariamente de un alto nivel de compromiso, codependencia positiva y comunicación efectiva.

También es de reconocer que, a pesar de proponer un trabajo cercano de la práctica docente, la falta de dominio técnico fue un contrapunto para la interacción virtual, por lo que fue necesario establecer mecanismos paralelos de apoyo, situación que debe considerarse para futuras experiencias de aplicación, a través de la disminución del trabajo encomendado a cada equipo, así como el fortalecimiento de la capacitación en el manejo de la aplicación virtual seleccionada.

Sobre la intervención didáctica se encontró que la observación realizada al grupo muestra dio cuenta de un efecto positivo en la intervención didáctica de los profesores, especialmente en las subcategorías de planeación didáctica, recursos didácticos y evaluación. De acuerdo con la información obtenida de la entrevista semiestructurada, los profesores refirieron como favorable contar con una planeación diseñada por otros profesores, a la cual realizaron ajustes de acuerdo con el nivel de desempeño del grupo, número de alumnos, recursos disponibles, entre otros aspectos. Para ello fue de gran importancia la integración del repositorio, el cual se constituyó como un bagaje de opciones al alcance de los profesores que fungió como una alternativa concreta de expandir sus concepciones sobre la enseñanza y el aprendizaje, al conocer nuevas y diversas formas de abordar los contenidos.

Desde la perspectiva de la ATP, como observadora externa, así como de la apreciación de los propios docentes, el resultado de trasladar al aula las propuestas surtió efectos positivos en el desenvolvimiento didáctico de los profesores. En esta línea se ratificó la pertinencia de proporcionar indicadores específicos para el diseño de las planeaciones didácticas, lo cual les facilitó la sistematización, bajo parámetros similares, en la autovaloración de su práctica pedagógica.

Sobre los procesos del agente promotor de la innovación, el papel de la ATP experimentó transformaciones en las diferentes etapas del proyecto. Pasó de fungir como intermediario de las disposiciones oficiales a convertirse en un gestor de la innovación. Ello implicó abandonar su zona de confort para adentrarse en una nueva forma de intervenir en el aprendizaje docente, en la cual desempeñó la organización, motivación y apoyo en algunos aspectos específicos a los equipos de trabajo, hasta el seguimiento del efecto de la CVA en las aulas.

No obstante, la ATP enfrentó cierta resistencia por parte de algunos profesores, quienes expresaron que el trabajo en la CVA podría representar mayor carga laboral; situación que representó un fenómeno hasta cierto punto inesperado, pero también comprensible. La resistencia por parte del profesorado es un fenómeno frecuente frente a un intento de cambio estructural, ya sea impulsado por los mismos docentes o por algún agente externo (en este caso desde la supervisión escolar). 
A partir de Monereo (2010), el origen de la resistencia es de carácter complejo y multifactorial. De esta manera puede generarse desde el terreno personal de los individuos al abandonar un área que ellos consideran de dominio hacia otra de vulnerabilidad; también puede partir del mismo terreno profesional, puesto que la cultura magisterial se ha caracterizado por brindar a los docentes limitados márgenes de actuación y decisión, o bien la resistencia se orienta sobre líneas institucionales tendientes a desvalorizar el conocimiento profesional docente.

El planteamiento de una CVA implica para toda estructura educativa la reformulación de actuaciones y dinámicas que no todos los docentes están dispuestos a asumir, por ejemplo, trabajar en equipo con otros docentes con quienes probablemente no tengan un contacto o buena relación personal, desenvolverse en una aplicación virtual con la que están poco familiarizados, además de exponer sus conocimientos y habilidades pedagógicas frente a otros. Estas son solo algunas de las incertidumbres inherentes a la propuesta de innovación que, aunque buscaba brindar mayor protagonismo a los profesores, también corrió el riesgo de interpretarse como un intento de reforma vertical al proceder de una autoridad educativa.

\section{Conclusiones}

Se ha expuesto la experiencia en torno una propuesta basada en las comunidades virtuales de aprendizaje para fortalecer la colaboración entre docentes en el mejoramiento de la intervención didáctica. Con base en la sistematización de la experiencia, con de resaltar algunas bondades y complejidades de aterrizar un modelo formativo de tal magnitud y alcance.

Gracias al manejo de instrumentos de investigación, se hallaron elementos relevantes al análisis de los alcances del entorno virtual en las distintas vertientes hacia las que se encuentra orientado. Se reconocieron valiosas contribuciones del aprendizaje en red hacia una formación del profesorado más auténtica través de la desfocalización del conocimiento, la interactividad y la reducción de los costos frente a cursos de enseñanza presenciales y unilaterales, aspectos en suma novedosos en el marco del modelo pedagógico de telesecundaria, cuya naturaleza solicita del profesorado un amplio dominio y movilización de saberes disciplinares, pedagógicos, curriculares y didácticos. Aunque también se sortearon algunos obstáculos, tales como la resistencia al cambio, la desmotivación, la impersonalidad, escasa cooperación y ausencia de contacto humano, elementos que, en la praxis, pueden incluso llegar a desmantelar el intento de innovación (Díaz, Urbano y Berea, 2013).

Por otra parte se identifica que el seguimiento investigativo del proyecto, basado principalmente en la investigación-acción, permitió la observación y sistematización focalizadas de la información concerniente a las categorías de análisis expuestas, empe- 
ro, también se reconocen áreas de oportunidad de carácter metodológico relacionadas principalmente con la profundización en los saberes docentes puestos en juego por parte del profesorado participante; la atención de esta variable alude necesariamente a métodos etnográficos en situaciones específicas de actuación (Rockwell, 2013), lo cual implica incorporación de otras técnicas, tales como grupos focales y entrevistas a profundidad, cuya aplicación resultó poco factible dada la brevedad de la intervención, no obstante se advierte su pertinencia para posibles recreaciones de la propuesta.

Así mismo se valora la acción del ATP cuya función, como cualquier otra, también es susceptible y demandante de evoluciones, develando la potencialidad de incidir en las prácticas docentes de una forma más auténtica y sustanciosa. En este contexto la asesoría técnica pedagógica también ha de reformularse permanentemente, pero sin perder de vista el sentido humano de la tarea docente. Al respecto, a pesar de que este campo del conocimiento aún enfrenta serias limitaciones metodológicas respecto a otras funciones históricamente consolidadas (Nieto, 2001), existen cuantiosas posibilidades de proyectar su impacto, ya sea presencial y/o virtualmente, siempre en función de las necesidades técnicas y pedagógicas identificadas.

Con base en los resultados obtenidos, se devela la pertinencia de atender necesidades de investigación concernientes principalmente a la interpretación del acontecer en el aula, sobre todo aquellas vinculadas a la esencialidad de la práctica docente reflexiva, con las finalidad de esclarecer las alternativas de impulsar la autonomía profesional del profesorado a través de la provisión de herramientas que le permitan encarar, desde una postura crítica, las diversas teorías, políticas, paradigmas (Zeichner y Liston, 1996), y en general la complejidad misma de la realidad escolar.

La reflexión profunda sobre la tarea educativa demanda una apertura constante a nuevos aprendizajes. Las herramientas virtuales por sí solas no son instrumentos o medios formativos, solo en la medida que son exploradas y experimentadas pueden perfilarse como alternativas en la construcción de conocimientos. Por ello la trascendencia de acercar a los profesores a una formación didáctica en ambientes de aprendizaje virtual, se comprende como antesala para integrar la tecnología tanto como estrategia de enseñanza como de crecimiento profesional (Villalpando, 2020). Las CVA pueden constituirse como espacios para la reflexión en tanto permitan una interacción profesional, abierta a nuevas interpretaciones de la tarea docente, para ello es fundamental que los ambientes virtuales dinamicen el flujo de nuevas formas de entender el actuar pedagógico, es decir, permitan teorizar la realidad para garantizar niveles cada vez más profundos de reflexión (Manen, 2004).

Finalmente, con miras a la institucionalización de las CVA como una alternativa de profesionalización, se perfila como área fértil de investigación la gestión de la innovación desde intervenciones epistémicas, apoyadas en grupos de trabajo inter- 
disciplinarios (Rojas, 2003), con la finalidad de construir una visión lo más completa posible de cómo se vivencian y germinan cada una de las etapas y procesos, así como aquellos requerimientos mínimos a considerar en la conformación de una CVA, cuyas experiencias apuntalen a la creación de culturas de innovación basadas en la socioformación virtual.

En definitiva, esta propuesta se reconoce como valiosa al condensar diversos tintes de innovación hacia el aprendizaje y la transformación de la práctica docente y de asesoría a través de la virtualidad. Aunque se identifican algunos contrapuntos de índole personal, profesional e institucional, se valora como significativo ahondar sobre estos en forma sistemática y proveer de mayor eficacia y solidez a las propuestas centradas en el campo de la formación docente desde un uso más significativo y eficaz de las tecnologías de la información, comunicación, conocimiento y aprendizaje digitales.

\section{REFERENCIAS}

Abero, L., Berardi, L., Capocasale, A., García, S., y Rojas, R. (2015). Investigación educativa: abriendo puertas al conocimiento. Montevideo, Uruguay: Clacso.

Álvarez-Gayou, J. L. (2003). Cómo hacerinvestigación cualitativa. Fundamentos y metodología. México: Paidós.

Boneu, J. M. (2007). Plataformas abiertas de e-learning para el soporte de contenidos educativos abiertos. RUSC. Universities and Knowledge Society Journal, 4(1), 3647. DOI: http://dx.doi.org/10.7238/rusc.v4i1.298.

Breda, A., Font, V., y Pino-Fan, L. R. (2018). Criterios valorativos y normativos en la didáctica de las matemáticas: el caso del constructo idoneidad didáctica. Bolema: Boletim de Educaşão Matemática, 32(60), 255-278.

Brito-Lara, M. (2017). La planeación didáctica de docentes de telesecundaria. Recuperado de: http://www.comie.org. $\mathrm{mx} /$ congreso/memoriaelectronica/v14/doc/1677. pdf.

Brousseau, G. (2007). Iniciación al estudio de la teoría de las situaciones didácticas/Introduction to study the theory of didactic situations: didáctico/didactic to algebra study (vol. 7). Buenos Aires: Libros del Zorzal.

Cabrera, F. C. (2005). Categorización y triangulación como procesos de validación del conocimiento en investigación cualitativa. Theoria, 14(1), 61-71. Recuperado de: https://www.redalyc.org/pdf/299/29900107. pdf.

Carbonell, J. (2000). La aventura de innovar: el cambio en la escuela. Madrid: Morata.
Castañeda, B. (2018). Una aproximación técnica y conceptual de la percepción de rúbricas de observación en el aula y el desempeño docente en las instituciones educativas del nivel de educación inicial del Distrito de Chamaca-Chumbivilcas, Cusco-2017.

Cruz-Abarca, L., Bazán-Ramírez, A., Taracena-Ruiz, E., y Castellanos-Simons, D. (2016). Configuración de la identidad profesional del asesor técnico pedagógico de educación básica. Diálogos sobre Educación, (14). Recuperado de: http://www.revistascientificas. udg.mx/index.php/DialogosRespaldo/article/ view/6941/5956\#.

Díaz, V. M., Urbano, E. R., y Berea, G. M. (2013). Hacia la determinación de posibles ventajas e inconvenientes de la formación online. Revista Docencia Universitaria, 14(1), 57-72. Recuperado de https:// revistas.uis.edu. co/index.php/revistadocencia/article/view/4224.

Echeverría, M. D. P. P., Municio, J. I. P., Rubiños, N. S., De la Cruz, M., Ortega, E. M., y Sanz, M. M. (2006). Nuevas formas de pensar la enseñanza y el aprendizaje: las concepciones de profesores y alumnos (vol. 12). Graó.

Eliott, J. (2000). Guía práctica para la investigaciónacción. En El cambio educativo desde la investigación acción (pp. 88- 111). Madrid: Morata.

Estrada, E. J. (2017). La telesecundaria en México. Orígenes y reformas. Dilemas Contemporáneos: Educación, Politica y Valores, 4(2). Recuperado de: http://www. dilemascontemporaneoseducacionpoliticayvalores. com/index.php/dilemas/article/view/150/740. 
Fullan, M., y Hargreaves, A. (1999). La escuela que queremos. México: SEP.

Gairín, J. (2006). Las comunidades virtuales de aprendizaje. Educar, 37, 41-64. Recuperado de: https://www. redalyc.org/articulo.oa?id $=3421 / 342130826004$.

García-Peñalvo, F. J. (2015). Mapa de tendencias en innovación educativa. Education in the Knowledge Society, 16(4), 6-23. Recuperado de: https:/ / www.redalyc.org/ articulo.oa?id $=5355 / 535554760001$.

Gehrig, R., Palacios, J., Blesa, B., Cobo, F., García, M., Muñoz, P., y Rodes, J. (2014). Guía de criterios básicos de calidad en la investigación cualitativa. España: UCAM.

Godino, J., Batanero, C., Rivas, H., y Arteaga, P. (2013). Componentes e indicadores de idoneidad de programas de formación de profesores en didáctica de las matemáticas. Revista Eletrônica de Educação Matemática, 8(1), 46-74. DOI: https://doi.org/10.5007/19811322.2013v8n1p46.

Grupal, E. D. L. D. C. (2015). Lista de cotejo, lista de control o check-list. Presentación I.

Hernández, R., Fernández-Collado, C., y Baptista, L. (2014). Metodología de la investigación. México: McGrawHill Interamericana.

Herrera, M. Á. (2006). Consideraciones para el diseño didáctico de ambientes virtuales de aprendizaje: una propuesta basada en las funciones cognitivas del aprendizaje. Revista Iberoamericana de Educación, 38(5), 1-20. DOI: https://doi.org/10.35362/rie3852623.

Johnson, D. W., Johnson, R. T., y Holubec, E. J. (1999). El aprendizaje cooperativo en el aula. Virginia, E.U.

Knowles, M. S., Holton III, E. F., y Swanson, R. A. (2006). Andragogia: el aprendizaje de los adultos. Oxford University.

Krichesky, G. J., y Murillo, F. J. (2018). La colaboración docente como factor de aprendizaje y promotor de mejora. Un estudio de casos. Educación XX1, 21(1), 135-155. Recuperado de: https://www.redalyc.org/ articulo.oa?id=706/70653466007.

LGE [Ley General de Educación] (2019). Diario Oficial de la Federación. México. Recuperado de: https:// dof.gob.mx/nota_detalle.php?codigo $=5573858 \& f e$ cha $=30 / 09 / 2019$.

LGSCMM [Ley General del Sistema para la Carrera de las Maestras y los Maestros] (2019). Diario Oficial de la Federación. México. Recuperado de: https:// dof.gob.mx/nota_detalle.php?codigo $=5573860 \& \mathrm{f}$ echa $=30 / 09 / 2019$.

Lucero, M. M. (2003). Entre el trabajo colaborativo y el aprendizaje colaborativo. Revista Iberoamericana de Educación, 33(1), 1-21. DOI: https://doi.org/10.35362/ rie3312923.

Manen, V. M. (2004). El tacto en la enseñanza. El significado de la sensibilidad pedagógica. Cap. 5 La práctica de la pedagogía. Paidós Ibérica, S.A.

Marín, V., Reche, E., y Maldonado, G. A. (2013). Ventajas e inconvenientes de la formación online. Revista Digital de Investigación en Docencia Universitaria, 7(1), 33-43. DOI: https://doi.org/10.19083/ridu.7.185.

Mercado, R. (1991). Los saberes docentes en el trabajo cotidiano de los maestros. Infancia y Aprendizaje, 14(55), 59-72.

Microsoft (2019). Le damos la bienvenida a Microsoft Teams. Recuperado de: https://docs.microsoft.com/es-es/ microsoftteams/teams-overview.

Monereo, C. (2010). ¡Saquen el libro de texto! Resistencia, obstáculos y alternativas en la formación de los docentes para el cambio educativo. Revista de Educación, (352), 586-597.

Nieto, C. (2001). Modelos de asesoramiento a organizaciones educativas. En Asesoramiento al centro educativo: colaboración y cambio en la institución (pp. 147-166). Octaedro.

Núñez, F. C., y Urquijo, A. Q. (2012). Importancia de la evaluación y autoevaluación en el rendimiento académico. Zona Próxima: Revista del Instituto de Estudios Superiores en Educación, (16), 96-104.

Pazos, M., Pérez, A., y Salinas, J. (2001). Comunidades virtuales: de las listas de discusión a las comunidades de aprendizaje. En Comunicación. Edutec'01. V Congreso Internacional de Tecnología, Educación y Desarrollo sostenible (pp. 17-19).

Quintana, A. (2006). Metodología de investigación científica cualitativa. En A. Quintana y W. Montgomery (eds.) Psicología tópicos de actualidad (pp. 65-73). Lima: UNMSM.

Rheingold, H. (1993). The virtual community: Finding commection in a computerized world. Addison-Wesley Longman.

Rivas, M. (2000). Innovación educativa: teoría, procesos y estrategias. España. 
Rivera Hernández, C. (2017). Propuesta de un sistema de formación especializada en supervisión educativa: análisis comparativo de 13 sistemas de supervisión escolar en el mundo. Innovación Educativa, 17(74), 165-179. Recuperado de: http://www.scielo.org. $\mathrm{mx} /$ scielo.php? script $=$ sci_arttext\&pid $=\mathrm{S} 1665$ 26732017000200165\&lng=es\&tlng $=$ es.

Rivera, L., y Martínez, A. (2015). La función de asesoría desde las politicas educativas y los actores: un análisis comparativo. Recuperado de: http://200.23.113.59:8080/handle123456789/1071.

Rockwell, E. (2013). El trabajo docente hoy: nuevas huellas, bardas y veredas. En XI Congreso Nacional de Investigación Educativa (pp. 437-473).

Rojas, R. (2003). Guía para realizar investigaciones sociales. México.

Salinas, P. I., Nevárez, M. O. T., y Torres, A. H. (2014). La planeación didáctica como factor determinante en la autoeficacia del maestro universitario. IE Revista de Investigación Educativa de la REDIECH, 5(9), 43-50. Recuperado de https://www.redalyc.org/ pdf/5216/521651961005.pdf.

Segovia, J. D. (2010). Comprender y redireccionar las prácticas de asesoría. Revista Iberoamericana ee Educación, (54), 65-83. Recuperado de: https://doi. org/10.35362/rie54054.

Senge, P. M. (2012). La quinta disciplina 9Ed: cómo impulsar el aprendizaje en la organización inteligente. Granica.

SEP [Secretaría de Educación Pública] (2019). Lineamientos generales para la operación del Servicio de Asesoría y Acompañamiento a las Escuelas en Educación Básica (SIS AAE).
Recuperado de: http:/ / cefdocente.edugem.gob.mx/ doctos/sate_lineamientos_generales.pdf.

SEP [Secretaría de Educación Pública] (2020). Agenda Digital Educativa (Ade.Mx). México. Recuperado de: https://infosen.senado.gob.mx/sgsp/gaceta/64/2/2020-02-05-1/assets/documentos/Agenda_Digital_Educacion.pdf.

Solís, M. E. R., Hernández, B. R. R., Téllez, L. S., Cuenca, P. O., y Guerrero, J. L. T. (2007). Las fases de la innovación educativa y la integración de la red responsable de la innovación: un caso ilustrativo para la profesionalización docente. Ponencia presentada en el Congreso Virtual Educa 2007. Brasil.

Tardif, M. (2004). Los saberes del docente y su desarrollo profesional (vol. 97). Narcea.

Vaillant, D. (2016). Trabajo colaborativo y nuevos escenarios para el desarrollo profesional docente.

Vaillant, D., y Marcelo, C. (2015). El A, B, C, D de la formación docente. Madrid: Narcea.

Villalpando, I. (2020). La escuela mexicana ante la pandemia: diagnóstico y escenarios posibles. Faro Educativo, Apunte de Política, (9).

Zeichner, K. M., y Liston, D. P. (1996). Historical roots of reflective teaching. En Reflective teaching. An introduction (pp. 8-18). Nueva Jersey, Lawrence Erlbaum Associates.

Zorrilla, M. (2004). La educación secundaria en México: al filo de su reforma. REICE. Revista Iberoamericana sobre Calidad, Eficacia y Cambio en Educación, 2(1), 0. Recuperado de: https://www.redalyc.org/articulo. oa?id $=55120106$.

Cómo citar este artículo:

Bonilla Santamaría, K., y Ferra Torres, G. E. (2021). Comunidades virtuales e innovación: propuestas desde la asesoría técnica pedagógica en la escuela telesecundaria. IE Revista de Investigación Educativa de la REDIECH, 12, e1102. doi: https://doi.org/10.33010/ie_rie_rediech.v12i0.1102. 